\title{
474834 - EPIDURAL ANALGESIA AND POSTOPERATIVE MORTALITY
}

\author{
Duminda Wijeysundera, MD FRCPC ${ }^{1}$, Peter Austin, PhD $^{2}$, Scott Beattie, $\mathrm{MD}^{1}$, \\ Janet Hux, MD MSc ${ }^{2}$, Andreas Laupacis, MD MSc ${ }^{3}$ \\ 1. Anesthesia, University Health Network, Toronto, ON, Canada \\ 2. Institute for Clinical Evaluative Sciences, Toronto, ON, Canada \\ 3. Keenan Research Centre, Li Ka Shing Knowledge Institute, St. Michael's \\ Hospital, Toronto, ON, Canada
}

Introduction: Although epidural anesthesia or analgesia has numerous benefits, including improved postoperative analgesia and attenuation of the surgical stress response, its effects on postoperative survival remain controversial. Published

randomized controlled trials are underpowered to detect plausible treatment effects. ${ }^{1,2}$ In addition, they often have limited generalizability. ${ }^{3}$ We therefore undertook a populationbased observational study to determine whether perioperative epidural anesthesia/analgesia is associated with improved 30-day survival after elective intermediate-to-high non-cardiac surgery.

Methods: Local REB approval was obtained for this study. We used population-based linked administrative databases to conduct a cohort study of 259,037 patients, aged $\geq 40$ years, who underwent selected elective intermediate-to-high risk non-cardiac surgical procedures between 1 April 1994 and 31 March 2004. Propensity-score methods were used to construct a matched-pairs cohort that resolved important baseline differences between patients who received epidural anesthesia/analgesia as opposed to those that did not. We then determined the association of epidural anesthesia/analgesia with 30-day mortality within these matched pairs. A pre-specified subgroup analysis was also performed, based on the anatomic location of the epidural catheter (thoracic versus lumbar).

Results: Of the 259,037 patients in the entire cohort, $22 \%(n=56,556)$ received perioperative epidural anesthesia/analgesia. The proportion of patients who received epidural anesthesia/analgesia rose gradually from 1994 (15\% of cases) to 2001 (26\% of cases), but subsequently decreased by 2004 (23\% of cases). The matched-pairs cohort consisted of 88,188 patients. Within the matched-pairs cohort, epidural anesthesia/analgesia was associated with a small reduction in 30-day mortality [1.74\% vs. $1.95 \%$; relative risk $0.89,95 \%$ confidence interval (CI) $0.81-0.98, \mathrm{P}=0.02$ ]. In the prespecified subgroup analysis, the effects of epidural anesthesia/analgesia did not vary with the anatomic level of the epidural catheter (thoracic level vs. lumbar level) $(P=0.20)$. Discussion: Perioperative epidural anesthesia/analgesia is associated with a small improvement in 30-day survival after elective intermediate-to-high risk non-cardiac surgery. This treatment effect should be interpreted cautiously. Despite the large sample size $(N=88,188)$, the estimate has borderline statistical significance $(P=0.02)$. In addition, absolute magnitude of this benefit is small ( $0.21 \%$ difference); this difference corresponds to a large number-needed-to-treat of 477 . Our study, therefore, does not provide compelling evidence for using epidural anesthesia/analgesia to improve overall postoperative survival. Nonetheless, our results support the safety of epidural 
anesthesia/analgesia when used in routine clinical practice for more proven indications, such as improving postoperative analgesia or preventing postoperative pulmonary complications.

References: 1. Lancet 2002 359: 1276-1282 2. Anesth Analg 2003 97: 919-920 3. Anesthesiology 1987 66:729-736 